\title{
Botulinum Toxin Injection: A Review of Injection Principles and Protocols
}

\author{
David E. Rapp, Alvaro Lucioni, Gregory T. Bales
}

Section of Urology, Department of Surgery, University of Chicago Hospitals, Chicago, Illinois, USA

\begin{abstract}
Despite the favorable outcomes seen using botulinum toxin (BTX) for voiding dysfunction using BTX, a standardized technique and protocol for toxin injection is not defined. We reviewed the current literature on intravesical BTX injection for DO (detrusor overactivity). Specific attention was placed on defining optimal injection protocol, including dose, volume, and injection sites. In addition, we sought to describe a standard technique to BTX injection.
\end{abstract}

Key words: botulinum toxin; detrusor overactive; techniques

Int Braz J Urol. 2007; 33: 132-41

\section{INTRODUCTION}

The introduction of botulinum toxin (BTX) within the field of urology offers an exciting new modality for the treatment of urologic disorders. The first reported urologic application of BTX injection was in the treatment of detrusor-sphincter dyssynergia (DSD) (1). In the subsequent years, the successful application of BTX has been reported in an increasing number of urologic disorders, including detrusor hypocontractility, sensory disorders, interstitial cystitis (IC), and benign prostatic hyperplasia (BPH) (2-5). To date, the most widespread urologic application of BTX has been in the treatment of urinary urgency and incontinence due to detrusor overactivity (DO). Accordingly, improved subjective and objective outcomes are demonstrated in numerous investigations using BTX injection in the treatment of both neurogenic and idiopathic detrusor overactivity populations (6-17).

Despite the initial success achieved via BTX injection in the treatment of voiding dysfunction, further improvement is necessary. Arguably, the most important immediate obstacle to the more successful widespread urologic utilization of BTX is the lack of a standardized technique for intravesical BTX administration. Published investigations to date have utilized varying doses, volumes, and injection sites/ numbers. Foremost, this variation makes systematic assessment of the safety and efficacy of BTX difficult. Further, it remains difficult to provide urologists seeking to incorporate BTX into their treatment armamentarium with a standardized protocol for administration. This article reviews the common protocols reported in the literature with specific focus on injection dose, distribution, and volume. Further, 
data is presented to help define related clinical issues, including duration of action, treatment onset, and benefit of repeat injection. Finally, we describe a standard technique for intravesical BTX injection. Due to the expanding number of urologic BTX applications (e.g. IC and BPH), this review will center on the most widespread application of BTX injection for DO.

\section{PROTOCOL FOR BOTULINUM TOXIN INJECTION}

\section{Injection Dose, Efficacy Outcomes}

A total injection dose of 100 to $400 \mathrm{U}$ (Botox ${ }^{\circledR}$ ) and 500 to $1000 \mathrm{SU}$ (Dysport ${ }^{\circledR}$ ) has been reported in published study to date. The vast majority of reported literature has used the Botox ${ }^{\circledR}$ preparation and, for this reason, the following discussion will focus on this preparation. Data using the Dysport ${ }^{\circledR}$ preparation is presented as available. Clinical experience suggests a toxin equivalency of $1 \mathrm{U}$ Botox ${ }^{\circledR}$ to 3.5-5 SU Dysport® (18).

The majority of published study has utilized a 300 U dose (6-17). Several studies are reported using a $200 \mathrm{U}$ dose and isolated study is described using both 100 and $400 \mathrm{U}$ doses $(7,8,11,16)$. In comparing the efficacy results of these studies, similar improvement is seen with respect to both subjective and objective outcomes. Outcome measures include incontinence episodes, quality of life indices, mean cystometric capacity, compliance, and decreased mean voiding pressures. Despite similar outcomes, definitive conclusions regarding the optimal dose remain difficult in the absence of study directly focused on dose-response outcomes.

Few individual studies have utilized variable dosing. In one of the first published investigations of BTX injection, Schurch and colleagues reported the use of 200 and $300 \mathrm{U}$ in the treatment of NDO (7). The authors reported that the administration dose was based on previous titration study demonstrating that this range was most likely to result in a complete blockade of acetylcholine at the detrusor level. Although a dose-response analysis was not formally conducted, the two patients failing to respond to treatment both received $200 \mathrm{U}$. As a result, the authors concluded that $300 \mathrm{U}$ might be the optimal dose for NDO.

The same group subsequently reported the first direct comparison of 200 versus $300 \mathrm{U}$ (8). In this prospective investigation, 59 patients with NDO were randomized to receive injection of 200 or $300 \mathrm{U}$ of BTX-A. Significant subjective and objective improvement was seen in the two study arms, including improved continence, bladder capacity, and maximum detrusor pressure. No difference in primary outcomes was demonstrated when comparing study arms. However, the authors caution that this outcome may have been affected by the small study sample size.

Kuo et al. recently reported a randomized comparison of 100,150 , and $200 \mathrm{U}$ in the treatment of refractory DO (19). Clinical and urodynamic outcomes were similar between the 150 and $200 \mathrm{U}$ groups, with those patients receiving $100 \mathrm{U}$ experiencing less favorable therapeutic results. Based on these data, the authors conclude that a $150 \mathrm{U}$ dose provides a satisfactory outcome with decreased adverse effects compared to the $200 \mathrm{U}$ dose (discussed below). As $300 \mathrm{U}$ is the most utilized dose to date, the failure to include this dose in the study comparison is a notable limitation.

Recently, Ruffion et al. investigated the benefit of 500 versus $1000 \mathrm{SU}$ of Dysport ${ }^{\circledR}$ in the treatment of NDO (20). Following toxin injection, 76\% of patients were found to be completely dry using both doses. Non-responders to $500 \mathrm{SU}$ also failed to achieve a response using a 1000 SU dose, suggesting that the overall efficacy rates are similar when using both doses. Of note, this report also describes an earlier experience using $300 \mathrm{SU}$, which had no clinical effect.

\section{Injection Dose, Duration Outcomes}

Histological evidence suggests that toxin injection is followed by a chemical dennervation, which is followed by re-sprouting of axons $(21,22)$. The timing of axonal re-sprouting is variable and is observed over a period of weeks to months. However, Haferkamp et al. found that only $3 / 7$ biopsy specimens demonstrated axonal sprouting at 9 months following 
injection (22). As symptom benefit has been generally shown to subside by this time point, this data suggests that duration of effect may not relate primarily to axonal sprouting.

Alternative data suggests that the local action of intra-detrusor BTX injection may effect a functional motor inhibition not associated with neuronal death. Further, BTX has recently been demonstrated to have inhibitory effects on additional neuronal populations (e.g. sensory, autonomic) and non-neuronal tissue (e.g. urothelium) (23-25). Apostolidis and associates demonstrated through immunohistochemistry study that overall neuronal density within bladder biopsy specimens was not significantly reduced at 4 and 16 weeks following toxin injection (26). However, a reduced expression of the sensory neuron receptors TRPV1 and $\mathrm{P} 2 \mathrm{X}_{3}$ was observed and corresponded with the clinical benefit seen in the patients. Recently, Khera et al. reported that BTX injection inhibited urothelial ATP release, suggesting that toxin injection may suppress purinergic sensory signaling through this action (27). Finally, while the muscular integrity is not altered following intra-detrusor injection, decreased bladder wall fibrosis is seen in patients following toxin injection when compared to those patients without toxin injection $(22,28)$. While a complete description of related research is beyond the scope of this review, this data is particularly important as the specific mechanism of toxin action may relate to the durability of clinical effect.

Irrespective of these factors, the clinical benefit of intradetrusor BTX injection appears to last at least six months. Shurch and colleagues reported a duration of at least nine months in their initial experience enrolling patients with NDO (7). Subsequent to this study, nearly all studies have concluded that the duration of action ranges from 6 to 10 months (6-17). Importantly, this duration has been found irrespective of treatment population (i.e. NDO vs. IDO) and Botox ${ }^{\circledR}$ dose used. The previously described dose comparison study by Shurch and associates demonstrated similar therapeutic benefit when comparing 200 and $300 \mathrm{U}$, which lasted through the study termination (8). However, the study duration of 24 weeks limits the conclusions that may be made with respect to injection duration. In contrast, Ruffion et al. concluded that 1000 SU Dysport ${ }^{\circledR}$ was associated with a significantly longer duration of action (10.4 vs. 4.8 months) when compared to $500 \mathrm{SU}$ (20). In those patients initially receiving $500 \mathrm{SU}$ who went on to receive repeat injections using $1000 \mathrm{SU}$, the duration increased consistent with those patients initially receiving $1000 \mathrm{SU}$. Certainly, further study is needed to better define the relation of injection dose and outcome duration. However, we believe that it is appropriate to counsel patients that the treatment effect may last at least six months.

\section{Injection Dose, Side Effects}

Certainly, a significant concern related to dose escalation lies in the potential side effects. To date, only one report evaluated overall adverse events utilizing a direct comparison of two Botox ${ }^{\circledR}$ doses. No difference was seen in overall adverse events between 200 versus $300 \mathrm{U}$ (8). Again, further large scale, direct comparison investigation is needed to better assess the side effect profile associated with varying doses. Nonetheless, some general conclusions may be drawn from the reported experiences using varying doses.

Foremost, significant side effects associated with BTX injection are uncommon. Hematuria and post-operative pain are the most common symptoms observed following toxin injection, however, no data exists to suggest a dose dependent nature to these effects. Given the paralytic nature of BTX, systemic effects are of significant theoretical concern. To date, no severe systemic complication (e.g. respiratory muscle weakness/paralysis) has been reported. Less severe systemic side effects, such as extremity weakness, are not reported in the vast majority of series reported to date. Del Popolo reported hypostenia with reduced supralesional muscle force in 5/61 patients undergoing $300 \mathrm{U}$ toxin injection for DO (29). These symptoms disappeared by four weeks following injection. Grosse et al. reported transient trunk and/ or extremity weakness in four patients (total six injections) following Dysport ${ }^{\circledR}$ injection using both a $750 \mathrm{SU}$ and $1000 \mathrm{SU}$ dosing (18). In these cases, the duration of muscle weakness ranged from two weeks 
to two months. Finally, Wyndaele et al. reported upper extremity weakness in 2 patients following intravesical injection of BTX (300 U Botox ${ }^{\circledR}, 1000$ SU Dysport $\left.{ }^{\circledR}\right)$, persisting in these cases for 90 days (30). While these reports represent isolated cases, it is important to note that no such events are seen using lower doses of both preparations (200 U Botox ${ }^{\circledR}, 500$ SU Dysport $\left.{ }^{\circledR}\right)$.

Certainly, urinary retention is a significant concern following toxin injection and may also be directly related to injection dose. Following intravesical BTX injection (300 U), Rajkumar et al. report an increased PVR in an IDO cohort (12). However, no incidence of urinary retention requiring catheterization was reported. Using a $300 \mathrm{U}$ dose, Kessler et al. reported de novo CIC in 9 patients (4 IDO, 5 NDO) due to a PVR greater than $150 \mathrm{~mL}$ (14). Popat and colleagues report de novo CIC in $69 \%$ of NDO patients $(300 \mathrm{U})$ as compared to $19 \%$ of those with IDO (200 U) (15). Finally, in the previously described comparison of 150, 200 and $250 \mathrm{U}$, Kuo et al. found that a dose-dependent increase in difficulty voiding and acute urinary retention (100 U: 0\%, $150 \mathrm{U}: 10 \%$, 200 U: 20\%) was seen over all doses (19). A separate investigation of NDO patients by the same author found that $30 \%$ of study participants required CIC (31). Of note, the majority of those patients requiring CIC were men. Despite these reports, no incidence of urinary retention is reported in the majority of investigation, making it difficult to reach definitive conclusions regarding the true incidence of urinary retention and its relation to dose escalation.

Despite the absence of conclusive data suggesting a higher incidence of adverse effects associated with $300 \mathrm{U}$, dose modification based on specific patient parameters has been reported in an attempt to reduce the risk of clinically significant PVR and/or urinary retention. Rackley et al. report the use of a $100 \mathrm{U}$ trial dose in patients with detrusor overactivity combined with urodynamic evidence of borderline contractility (17). These authors also use this trial dose in patients of advanced age, given evidence demonstrating advanced age to predict for hypocontractile bladder conditions. Smith and colleagues report the use of a greater number of injections in patients with NDO as compared to those with IDO or IC (16). This approach may be particularly effective in this subset of patients, who perform CIC but remain incontinent as a result of detrusor overactivity. Accordingly, detrusor hypocontractility may actually be a desired effect in these patients.

\section{Injection Volume}

Published investigation to date has generally used an injection volume ranging from $0.1 \mathrm{~mL}$ to 0.5 $\mathrm{mL} /$ injection site. More recently, injection techniques using a larger injection volumes have been discussed (32). Larger dilution volumes have been shown to result in increased gastrocnemius muscle relaxation in an animal model (33). Theoretically, it is possible that larger dilution volumes will result in greater suburothelial diffusion, thereby allowing for toxin action on a larger surface area of muscle. However, no evidence has been presented to suggest that increased dilution volume used during intravesical injection BTX will result in superior clinical outcomes. In contrast, larger volumes may have the deleterious effect of increasing the potential for serosal extravasation. Further, as BTX administration is more frequently performed in the outpatient clinical setting, larger volumes may also result in increased patient discomfort.

\section{Injection Distribution}

In general, toxin injection is performed using 20-40 evenly distributed intramural injection sites. These sites include the bladder base and posterolateral walls of the bladder. As the wall of the bladder dome is the thinnest bladder region and lies in an intraperitoneal position, this area is avoided to prevent inadvertent bowel injury. Figure-1 illustrates the injection template used by Smith et al. In contrast to this uniform distribution pattern, other authors use an injection template concentrated over the posterior bladder wall only (34).

Perhaps the greatest question related to optimal injection template involves the decision to include or spare the trigone. The initial report of intravesical toxin injection described a trigone-sparing injection distribution (7). This decision to avoid the trigone was multifactorial, including a desire to avoid inducing reflux to the upper tracts. In addition, it was believed that injection of the dense trigonal innervation 
A

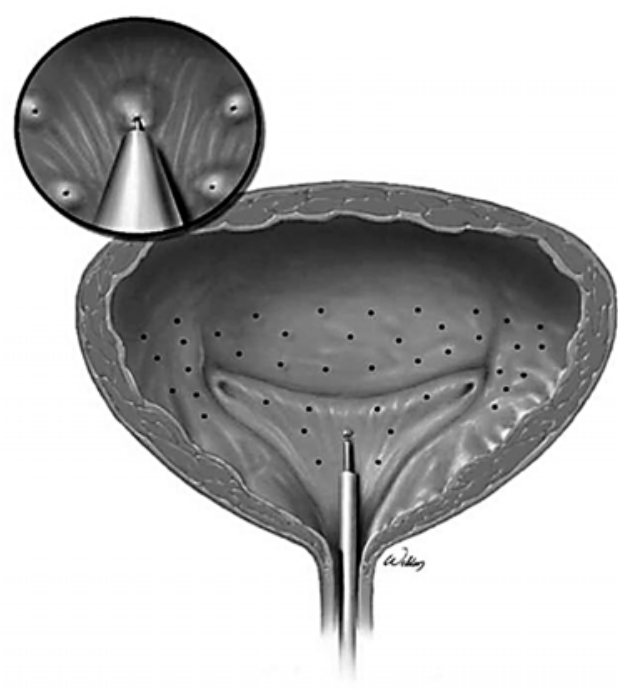

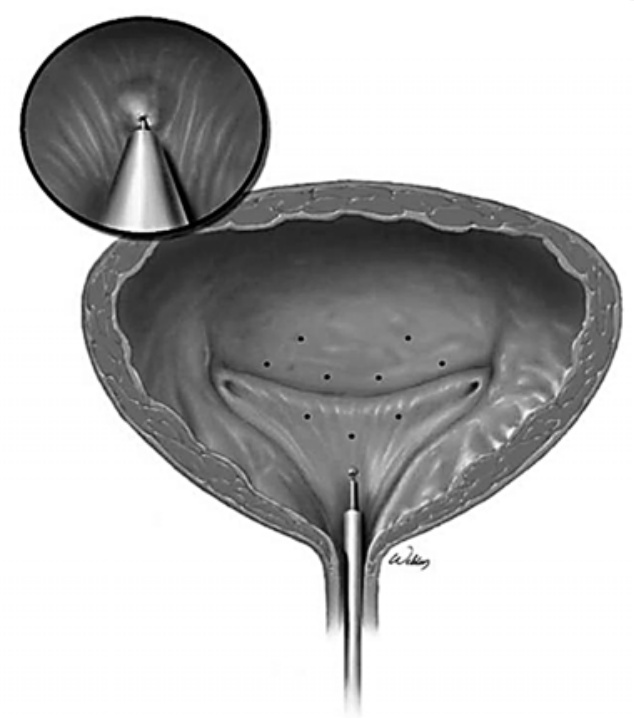

Figure 1 - Smith et al., botulinum toxin injection protocol. A) Neurogenic detrusor overactivity protocol. B) Non-neurogenic detrusor overactivity (Reprinted with permission from Elsevier Inc, Urology, Smith CP et al., ref. 16).

from both sensory, adrenergic, and non-cholinergic pathways might complicate the efficacy analysis of a cholinergic blockade. Subsequent investigations have predominantly utilized trigone-sparing injections. Whether these protocols were adapted based on similar concerns, simply a lack of other protocols to define trigonal inclusion, or for other reasons is unclear.

Given the concerns raised by Schurch et al., it was indeed reasonable for early investigators to spare the trigone in the absence of persuasive evidence to support trigonal inclusion. However, a significant amount of subsequent basic and clinical research has suggested that sensory neuron dysfunction may actually contribute to the pathophysiology of detrusor overactivity, sensory urgency, and IC (35-37). In addition, increasing evidence suggests that botulinum toxin inhibits both sensory neuron actions and the release of sensory neuropeptides from adjacent cell types (e.g. urothelium) that may contribute to sensory signaling $(23,24,27)$. Finally, BTX has also been show to decrease gene expression associated with bladder inflammation, suggesting a possible role in an even wider spectrum of pain-predominant disorders (e.g. IC) (38). Combined, these data would suggest a possible benefit to including the trigone in the injection distribution.

Two recent studies report successful outcomes utilizing a BTX injection with trigonal inclusion (16,17). However, no direct comparison was made with patients receiving trigone-sparing injections. Although routine post-operative voiding cystourethrogram (VCUG) was not performed to rule out the possibility of iatrogenic reflux, neither study reported postoperative urinary tract infection based on urinalysis and symptom presentation.

At the University of Chicago, we conducted a pilot study to assess the subjective benefit of trigonalinclusion during BTX injection (39). A total of 40 patients with $\mathrm{OAB}$ refractory to anticholinergic treatment underwent trigone or trigone-sparing injection of BTX-A (300 U). Our trigonal-inclusion protocol comprised 30 evenly distributed injections (10 U/injection site), with two injections being placed in the trigonal region (Figure-2). A statistically significant improvement in UDI and IIQ symptom scores was 


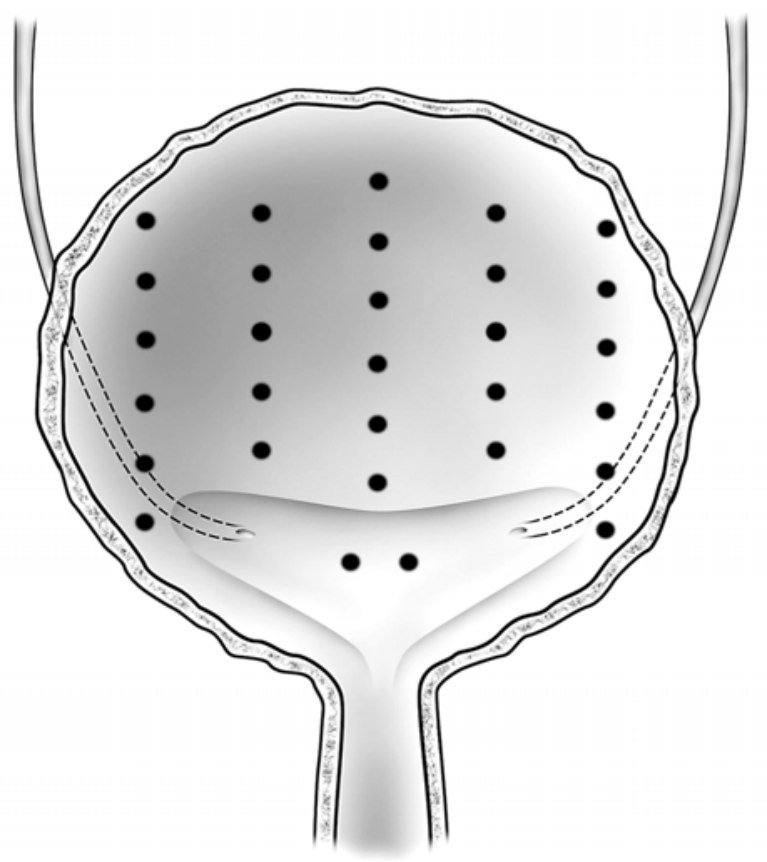

Figure 2 - University of Chicago botulinum toxin injection protocol (Reprinted with permission from Elsevier Inc, Urology, Rapp DE et al., ref. 13).

seen at 3-week and 6-month follow-up in both groups. However, no difference between the treatment arms was found. Notably, the trigonal injection number was arbitrary and it is possible that a greater number of injections within the trigonal region would have improved patient outcome. Despite these findings, further investigation is needed to determine whether trigonal injection is associated with improved urodynamic outcomes or may be more appropriately used in patients with isolated sensory and/or pain complaints. Further, in patients with pain- or sensorypredominant symptomatology, it may be possible that a trigone-only injection template may be sufficient to provide clinical benefit.

\section{CLINICAL ISSUES RELATED TO BTX INJECTION}

\section{Treatment Onset}

Few investigations define the exact onset of treatment response given the available literature. When reported, most investigations define treatment onset based on subjective response, introducing significant interpatient variation and recall bias. In contrast, objective outcomes, as demonstrated by urodynamic evaluation, are often not performed until 4 to 6 weeks following therapy. For these reasons, it is often difficult to define precise treatment onset.

Smith et al. reported that maximal efficacy was seen between 7 and 30 days following intradetrusor injection of BTX (16). Time to maximal efficacy was defined using patient interview conducted via telephone consultation or during clinic visit. In our investigation of 35 patients undergoing bladder injection of BTX for treatment of OAB, patient questionnaires were used to evaluate time to first and time to maximal symptom improvement (13). Responders reported first noting an improvement to their symptoms at a range of 1 to 14 days (mean 5.3) postoperatively and described reaching the maximal symptom improvement at 2 to 20 days (mean 8.3) postoperatively.

\section{Repeat Injection}

Two investigations have specifically evaluated the efficacy of BTX in patients undergoing repeat injection. Grosse and colleagues reported 66 patients undergoing repeat BTX injection (Botox ${ }^{\circledR}, 300 \mathrm{U}$, Dysport $\AA, 750 \mathrm{SU})$ in the treatment of neurogenic urinary tract dysfunction (40). All patients underwent one repeat injection, with a portion undergoing as many as six repeat injections. No difference was seen when comparing the difference between injection intervals. Major improvement in subjective satisfaction was seen in $71 \%$ of patients undergoing repeat injection and was comparable to the $74 \%$ rate observed following the initial injection. Urodynamic improvement in cystometric capacity and reflex volume were seen through the measured endpoint of injection 3.

Repeat injection is also reported in other studies. Smith et al. report 27 patients undergoing repeat injection at intervals of 6 months or longer (16). The authors comment that repeat injections usually lasted longer than the initial injection, with some patients having a durable response greater than 1 year. However, no specific data regarding repeat injection is provided in this report. Ruffion et al. report repeat Dysport ${ }^{\circledR}$ 
injection (500 versus $1000 \mathrm{SU}$ ) in 36 patients (20). In those patients receiving an initial dose of $1000 \mathrm{SU}$, there have been no treatment failures. All patients receiving a second injection revealed improved symptoms.

Based on these data, it appears that the efficacy of BTX injection continues in the majority of patients undergoing repeat injection. Undoubtedly, however, a small percentage of patients will fail repeat injection. Multiple toxin injection may cause resistance and associated treatment failure, with the development of anti-toxin antibodies being proposed as one potential etiology of resistance (41). This data served as one rationale for the investigation of BTX$B$ in the treatment of urologic disorders. The use of BTX-B for DO has been reported in de novo patients, those with documented BTX-A resistance, and in a cross-over investigation protocol (42-46). Subjective and objective improvement was seen in all studies. However, the short duration of effect (ranged six weeks to six months) suggests that the use of BTX-B may be most appropriate for patients initially failing BTX-A injection. Currently it is unclear which patients are likely to respond to repeat injection, which criteria should be used to time reinjection, and the role that BTX-B will play in this treatment algorithm.

\section{TECHNIQUE FOR INTRAVESICAL BOTULINUM TOXIN INJECTION}

\section{Bladder Injection Technique}

BTX injection is performed at our center using intravenous sedation. Toxin injection using local anesthesia is also reported and is associated with minimal discomfort. Rackley et al. report The Cleveland Clinic Foundation injection protocol, in which $100 \mathrm{~mL}$ of $2 \%$ lidocaine solution is instilled into the bladder with a 15 to 20 minute dwell time (17). More experience using BTX under local anesthesia may allow for cost reduction, avoidance of anesthetic risks, and injection in the clinic setting. All patients receive perioperative antibiotics.

Intravesical injection of BTX is performed by first diluting the toxin to the desired concentration. Botox is preserved in a vacuum-dried formulation, with each vile containing 100 units. At our institution, each vile of Botox ${ }^{\circledR}$ is diluted using $1 \mathrm{~mL}$ of preservativefree saline, yielding 10 units per $0.1 \mathrm{~mL}$ for injection at each site. The entire dilution is then drawn into a $1 \mathrm{~mL}$ syringe. A total of two or three vials are used, dependent on desired total dose. As excessive movement can decrease the potency of the toxin through disruption of its disulfide bonds, care is taken to avoid shaking during toxin preparation (47).

A variety of cystoscopic equipment has been used to perform BTX injection. At our institution, we use a rigid $21 \mathrm{~F}$ cystoscope and a collagen injection needle inserted through the endoscopic working port. Alternatively, a flexible cystoscope can be used and may be better suited for injection in the clinic setting, especially with the male patient. In this setting, a longer injection needle may be required and can be combined with the use of a sheath to stabilize the needle for ease during injection. Finally, both reusable and disposable injection needles may be used, however, the long-term cost associated with disposable needles must be considered.

BTX injection is initiated with standard cystoscopy in the dorsal lithotomy position. Following entry into the bladder with the cystocope, the needle tip is observed under direct vision. As the needle sheath volume approximates $0.5 \mathrm{~mL}$, priming is required. Accordingly, $0.5 \mathrm{~mL}$ of BTX is injected into the needle prior to insertion into the detrusor muscle. Visual confirmation of a sufficient priming dose is provided by observing for cessation of air bubble flow from the needle tip.

The bladder wall is then injected with BTX, divided among evenly distributed intramural injection sites. In male patients, a longer injection needle may be used when necessary. Twenty to 30 evenly distributed intra-detrusor injections are generally administered based on the specific protocol used (see previous discussion). Our injection technique involves the creation of a submucosal bleb, allowing for action on the underlying detrusor muscle. This technique allows for visual confirmation of the insertion depth and diffusion along the suburothelial space. Other authors attempt direct needle insertion and toxin injection within the detrusor muscle itself. When using this technique, care must be taken to avoid the risk of 
inserting the needle through the bladder serosa, with resultant toxin extravasation and risk to neighboring pelvic structures. Finally, BTX mixed with methylene blue is described to aid in identifying injection sites and to ensure a uniform distribution (34). However, this technique is not advocated by the manufacturers of Botox ${ }^{\circledR}$ due to the unknown interactions between these two agents. As a result of sheath priming, the final $0.5 \mathrm{~mL}$ of toxin are injected by flushing the sheath with a fourth $1 \mathrm{~mL}$ syringe containing $0.5 \mathrm{~mL}$ of saline.

\section{CONCLUSION}

The introduction of BTX injection offers a promising treatment for a variety of urologic disorders. Toxin injection may be easily performed using standard cystoscopic equipment in the outpatient or clinic setting. The greatest clinical experience is reported using 200 and $300 \mathrm{U}$ Botox ${ }^{\circledR}$. Available data suggest that clinical efficacy, duration, and the side effect profile is similar at these doses. While more data is needed, in the absence of conclusive evidence suggesting improved clinical outcomes using $300 \mathrm{U}$, the expense of BTX would support the use of a 200 $\mathrm{U}$ dose. Much less data is available regarding clinical outcomes using the Dysport ${ }^{\circledR}$ preparation. Isolated reports support that efficacy is similar when using a dosing range of 500 to $1000 \mathrm{SU}$. However, these same reports suggest that $1000 \mathrm{SU}$ may be associated with a longer duration of action, but increased risk of systemic side effects. While this data may suggest that $750 \mathrm{SU}$ is the optimal dose, no definitive data is present to support this conclusion.

A variety of injection volumes have been used, demonstrating similar efficacy and tolerability. However, no investigation has specifically compared variable volumes in the setting of one injection dose. Injection duration extends six to ten months in the majority of study. Data suggests that repeat injection is successful in the vast majority of initial responders, irrespective of preparation used (Botox ${ }^{\circledR}$ or Dysport $($ ). More experience is needed to precisely define the optimal protocol for BTX with respect to therapeutic outcomes and adverse effects.

\section{CONFLICT OF INTEREST}

\author{
None declared.
}

\section{REFERENCES}

1. Dykstra DD, Sidi AA, Scott AB, Pagel JM, Goldish GD: Effects of botulinum A toxin on detrusor-sphincter dyssynergia in spinal cord injury patients. J Urol. 1988; 139: 919-22.

2. Kuo HC: Effect of botulinum a toxin in the treatment of voiding dysfunction due to detrusor underactivity. Urology. 2003; 61: 550-4.

3. Zermann DH, Ishigooka M, Schubert J, Schmidt RA: Trigonum and bladder base injection of botulinum toxin A (BTX) in patients with severe urgencyfrequency-syndrome refractory to conservative medical treatment and electrical stimulation. Neurourol Urodyn. 2001; 20: 412-3, Abstract.

4. Smith CP, Radziszewski P, Borkowski A, Somogyi GT, Boone TB, Chancellor MB: Botulinum toxin a has antinociceptive effects in treating interstitial cystitis. Urology. 2004; 64: 871-5

5. Kuo HC: Prostate botulinum A toxin injection-an alternative treatment for benign prostatic obstruction in poor surgical candidates. Urology. 2005; 65: 670-4.

6. Bagi P, Biering-Sorensen F: Botulinum toxin A for treatment of neurogenic detrusor overactivity and incontinence in patients with spinal cord lesions. Scand J Urol Nephrol. 2004; 38: 495-8.

7. Schurch B, Stohrer M, Kramer G, Schmid DM, Gaul G, Hauri D: Botulinum-A toxin for treating detrusor hyperreflexia in spinal cord injured patients: a new alternative to anticholinergic drugs? Preliminary results. J Urol. 2000; 164: 692-7.

8. Schurch B, de Seze M, Denys P, Chartier-Kastler E, Haab F, Everaert K, et al.: Botulinum toxin type a is a safe and effective treatment for neurogenic urinary incontinence: results of a single treatment, randomized, placebo controlled 6-month study. J Urol. 2005; 174 : 196-200.

9. Reitz A, Stohrer M, Kramer G, Del Popolo G, ChartierKastler E, Pannek J, et al.: European experience of 200 cases treated with botulinum-A toxin injections into the detrusor muscle for urinary incontinence due to neurogenic detrusor overactivity. Eur Urol. 2004; 45: 510-5.

10. Schulte-Baukloh H, Michael T, Sturzebecher B, Knispel $\mathrm{HH}$ : Botulinum-a toxin detrusor injection as a novel 
approach in the treatment of bladder spasticity in children with neurogenic bladder. Eur Urol. 2003; 44: 139-43.

11. Hajebrahimi S, Altaweel W, Cadoret J, Cohen E, Corcos $\mathrm{J}$ : Efficacy of botulinum-A toxin in adults with neurogenic overactive bladder: initial results. Can J Urol. 2005; 12: 2543-6.

12. Rajkumar GN, Small DR, Mustafa AW, Conn G: A prospective study to evaluate the safety, tolerability, efficacy and durability of response of intravesical injection of botulinum toxin type A into detrusor muscle in patients with refractory idiopathic detrusor overactivity. BJU Int. 2005; 96: 848-52.

13. Rapp DE, Lucioni A, Katz EE, O'Connor RC, Gerber GS, Bales GT: Use of botulinum-A toxin for the treatment of refractory overactive bladder symptoms: an initial experience. Urology. 2004; 63: 1071-5.

14. Kessler TM, Danuser H, Schumacher M, Studer UE, Burkhard FC: Botulinum A toxin injections into the detrusor: an effective treatment in idiopathic and neurogenic detrusor overactivity? Neurourol Urodyn. 2005; 24: 231-6.

15. Popat R, Apostolidis A, Kalsi V, Gonzales G, Fowler CJ, Dasgupta P: A comparison between the response of patients with idiopathic detrusor overactivity and neurogenic detrusor overactivity to the first intradetrusor injection of botulinum-A toxin. J Urol. 2005; 174: 984-9.

16. Smith CP, Nishiguchi J, O'Leary M, Yoshimura N, Chancellor MB: Single-institution experience in 110 patients with botulinum toxin A injection into bladder or urethra. Urology. 2005; 65: 37-41.

17. Rackley RR, Frenkl TL, Abdelmalak JB: Botulinum toxin: the promise of therapy for complex voiding dysfunction. Cont Urol. 2005; 38-52.

18. Grosse J, Kramer G, Tong TQT, Stoher M, Jakse G: Efficacy, dosage, and safety of Dysport English botulinum toxin A in sever neurogenic detrusor overactivity. Eur Urol. 2005; suppl. 4: Abst \#405.

19. Kuo HC: Comparison of the therapeutic effects of suburothelial injection of botulinum A toxin at different doses for refractory detrusor overactivity. J Urol. 2006; 175: 113 (A347).

20. Ruffion A, Capelle O, Paparel P, Leriche B, Leriche A, Grise P: What is the optimum dose of type A botulinum toxin for treating neurogenic bladder overactivity? BJU Int. 2006; 97: 1030-4.

21. de Paiva A, Meunier FA, Molgo J, Aoki KR, Dolly JO: Functional repair of motor endplates after botulinum neurotoxin type A poisoning: biphasic switch of synaptic activity between nerve sprouts and their parent terminals. Proc Natl Acad Sci U S A. 1999; 96 : 3200-5.

22. Haferkamp A, Krengel U, Reitz A, Grosse J, Kramer G, Schumacher S, et al.: Are botulinum-A toxin injections into the detrusor of patients with neurogenic detrusor overactivity safe? Ultrastructural data of detrusor biopsies. Neurourol Urodyn. 2003; 22: 499-500.

23. Rapp DE, Turk KW, Bales GT, Cook SP: Botulinum toxin type a inhibits calcitonin gene-related peptide release from isolated rat bladder. J Urol. 2006; 175: 1138-42.

24. Chuang YC, Yoshimura N, Huang CC, Chiang PH, Chancellor MB: Intravesical botulinum toxin a administration produces analgesia against acetic acid induced bladder pain responses in rats. J Urol. 2004; 172: 1529-1532.

25. Sampaio FJ: Effect of botulinum toxin A on the autonomic nervous system of the rat lower urinary tract. Int Braz J Urol. 2003; 29: 176-8.

26. Apostolidis A, Popat R, Yiangou Y, Cockayne D, Ford AP, Davis JB, et al.: Decreased sensory receptors P2X3 and TRPV1 in suburothelial nerve fibers following intradetrusor injections of botulinum toxin for human detrusor overactivity. J Urol. 2005; 174: 977-82; discussion 982-3.

27. Khera M, Somogyi GT, Kiss S, Boone TB, Smith CP: Botulinum toxin A inhibits ATP release from bladder urothelium after chronic spinal cord injury. Neurochem Int. 2004; 45: 987-93.

28. Comperat E, Reitz A, Delcourt A, Capron F, Denys P, Chartier-Kastler E: Histologic Features in the Urinary Bladder Wall Affected from Neurogenic OveractivityA Comparison of Inflammation, Oedema and Fibrosis With and Without Injection of Botulinum Toxin Type A. Eur Urol. 2006; 50: 1058-64.

29. Del popolo G: Botulinum-A toxin in the treatment of detrusor hyperreflexia. Neururol Urodyn. 2001; 20: 522.

30. Wyndaele JJ, Van Dromme SA: Muscular weakness as side effect of botulinum toxin injection for neurogenic detrusor overactivity. Spinal Cord. 2002; 40: 599-600.

31. Kuo HC: Clinical effects of suburothelial injection of botulinum A toxin on patients with nonneurogenic detrusor overactivity refractory to anticholinergics. Urology. 2005; 66: 94-8.

32. Smith CP, Chancellor MB: Emerging role of botulinum toxin in the management of voiding dysfunction. J Urol. 2004; 171: 2128-37.

33. Kim HS, Hwang JH, Jeong ST, Lee YT, Lee PK, Suh YL, et al.: Effect of muscle activity and botulinum toxin 
dilution volume on muscle paralysis. Dev Med Child Neurol. 2003; 45: 200-6.

34. Mahajan ST, Kenton K, Brubaker L: Cystoscopic intradetrusor botulinum A toxin injection for the treatment of detrusor overactivity incontinence. J Urol. 2006; 175: 539, Abst \#1672.

35. Ray FR, Moore KH, Hansen MA, Barden JA: Loss of purinergic $\mathrm{P} 2 \mathrm{X}$ receptor innervation in human detrusor and subepithelium from adults with sensory urgency. Cell Tissue Res. 2003; 314: 351-9.

36. Chuang YC, Fraser MO, Yu Y, Chancellor MB, de Groat WC, Yoshimura N: The role of bladder afferent pathways in bladder hyperactivity induced by the intravesical administration of nerve growth factor. J Urol. 2001; 165: 975-9.

37. Szallasi A, Fowler CJ: After a decade of intravesical vanilloid therapy: still more questions than answers. Lancet Neurol. 20021;1: 167-72.

38. Vemulakonda VM, Somogyi GT, Kiss S, Salas NA, Boone TB, Smith CP: Inhibitory effect of intravesically applied botulinum toxin $\mathrm{A}$ in chronic bladder inflammation. J Urol. 2005; 173: 621-4.

39. Lucioni A, Rapp DE, Gong EM, Fedunok P, Bales GT: Botulinum toxin type Ainjection for overactive bladder: trigone versus trigone-sparing injection. (In Press).

40. Grosse J, Kramer G, Stohrer M: Success of repeat detrusor injections of botulinum a toxin in patients with severe neurogenic detrusor overactivity and incontinence. Eur Urol. 2005; 47: 653-9.
41. Doellgast GJ, Brown JE, Koufman JA, Hatheway CL: Sensitive assay for measurement of antibodies to Clostridium botulinum neurotoxins A, B, and E: use of hapten-labeled-antibody elution to isolate specific complexes. J Clin Microbiol. 1997; 35: 578-83.

42. Dykstra DD, Pryor J, Goldish G: Use of botulinum toxin type B for the treatment of detrusor hyperreflexia in a patient with multiple sclerosis: a case report. Arch Phys Med Rehabil. 2003; 84: 1399-400.

43. Dykstra D, Enriquez A, Valley M: Treatment of overactive bladder with botulinum toxin type B: a pilot study. Int Urogynecol J Pelvic Floor Dysfunct. 2003; 14: 424-6.

44. Reitz A, Schurch B: Botulinum toxin type B injection for management of type A resistant neurogenic detrusor overactivity. J Urol. 2004; 171: 804; discussion 804-5.

45. Pistolesi D, Selli C, Rossi B, Stampacchia G: Botulinum toxin type B for type A resistant bladder spasticity. J Urol. 2004; 171: 802-3.

46. Ghei M, Maraj BH, Miller R, Nathan S, O'Sullivan C, Fowler CJ, et al.: Effects of botulinum toxin B on refractory detrusor overactivity: a randomized, double-blind, placebo controlled, crossover trial. J Urol. 2005; 174: 1873-7.

47. Smaldone MC, Chancellor MB: Bladder or urethra injection of botulinum toxin type A: one institution's experience. Am J Urol Rev. 2005; 3: 422-426. $\overline{\text { Accepted: }}$

September 20, 2006

\author{
Correspondence address: \\ Dr. David E. Rapp \\ Department of Surgery, Section of Urology \\ University of Chicago Pritzker School of Medicine \\ 5841 S. Maryland Avenue, MC 6038 \\ Chicago, Illinois, 60637, USA \\ Fax: + 1773 702-1001 \\ E-mail:derapp@yahoo.com
}

